\section{Sixth European Conference on Clinical Aspects and Treatment of HIV Infection, Hamburg, Germany, 11-15 October 1997}

The conference was organised under the auspices of the European AIDS Clinical Society. The current president of the society is Nathan Clumeck of Belgium. In all, 2900 delegates attended.

Material presented suggested that eradication of virus from infected patients, even in those treated aggressively at a very early stage, is unlikely to be feasible with currently available agents.

David Ho provided an update on his cohort of acute seroconverters who received early aggressive antiretroviral treatment (plenary 3 ). At 28 months viral load remained undetectable in those patients who remained compliant with the drugs and virus was also not detected in seminal fluid, cerebrospinal fluid, or lymphoid tissue from the gut, tonsils, and lymph nodes. However, when multiple samples were analysed active RNA expression was found in a small number of cells suggesting continued, albeit low level, replication. Ho postulated that it may be possible to activate these semidormant cells using a cytokine cocktail allowing them to be destroyed subsequently with conventional antiretroviral agents. Those who received early therapy also demonstrated an attenuated cytotoxic $\mathrm{T}$ cell response and reduced gp120 antibody response suggesting that therapy will need to be continued long term.

The effect of triple antiretroviral therapy on lymphoid tissue was also assessed by Sven Danner in patients with established HIV infection (plenary 4). In this advanced disease group (CD4 count $<50 / \mathrm{ml}$ and viral load > 30000 cells $/ \mathrm{ml})$ the combination of zidovudine (ZDV), lamivudine (3TC), and ritonavir produced a $2 \frac{1}{2} \log$ reduction in lymph tissue viral burden associated with undetectable plasma viral load in almost all patients. Proviral DNA remained detectable in all patients.

Data presented at the meeting further added to the belief that HIV-RNA plasma viral load ( $p V L)$ is a useful surrogate marker for assessing the response to antiretroviral therapy.

In plenary session 2 Dr $M$ Elashoff presented the results of an exercise carried out by the Food and Drug Administration in which they looked at all the clinical trials to date which have provided information on this issue. They concluded that greater clinical benefit was predicted by (1) a greater decrease in $\mathrm{pVL}$ from the (pretreatment) baseline, with anything more than a $0.5 \log$ fall predicting some benefit; (2) the lower the pVL nadir, with no sign of a threshold below which no additional benefit would be seen; (3) the longer the duration of suppression of pVL below baseline (the "area under the curve" of the pVL was also predictive).

Virological data from the Delta trial analysing the role of HIV viral load as a surrogate marker for subsequent disease progression were presented by Dr Babiker (oral abstract no 103). He brought into question the role of viral load as a surrogate measure by demonstrating that it significantly overestimates the subsequent treatment effect. Also of concern was the variation observed in the strength of the association between changes in viral load and clinical outcome between different treatment arms in the trial-viral load was a better predictor of clinical outcome in the ZDV arm than the ZDV/ didanosine or $\mathrm{ZDV} /$ zalcitabine arms.

However, in another presentation an analysis of virology from the CAESAR trial found that $100 \%$ (with a lower $95 \%$ confidence interval of $56 \%$ ) of the clinical benefit seen in the patients receiving 3TC was explained by the observed changes in pVL and CD4 (oral abstract no 207). The difference may result from the longer observation time in the Delta trial.

Dr J Montaner presented virological data on 150 patients from the INCAS study showing that the duration of $\mathrm{pVL}$ suppression was greater both (a) the lower the nadir attained; (b) the shorter the time taken to reach this nadir (oral abstract no 105). Patients with nadirs below 20 cells $/ \mathrm{ml}$ had significantly longer virological suppression than those who did not. Importantly, patients with nadirs between 20 and 400 cells $/ \mathrm{ml}$ did no better than patients who never went below 400 implying that suppression below 20 should be the goal of therapy which would require clinicians to have access to the more sensitive assays that are now available.

Data were presented from the Avanti-2 trial which compared ZDV + 3TC with ZDV $+3 \mathrm{TC}+$ indinavir in therapy naive patients (oral abstract no 211). Not surprisingly, the triple therapy group showed better pVL and
CD4 cell responses. In patients with baseline pVL less than 20000 cells/ml suppression below 500 was not too different in the two arms but there was a more pronounced advantage for triple therapy in reducing $\mathrm{pVL}$ below 20, suggesting that double nucleoside analogue regimes cannot result in profound suppression of viral replication even in naive patients with relatively low initial viral loads. Furthermore, even triple therapy achieved suppression below 20 in only $40 \%$ of patients with baseline pVL above 20000 .

The message that double nucleoside analogue regimens are probably inadequate was also supported by a retrospective review of 245 patients in British Columbia who received such treatment first line: $70 \%$ of the subgroup with baseline pVL less than 20000 cells $/ \mathrm{ml}$ failed to achieve suppression below 50 on more than one occasion (oral abstract no 111).

The role of interleukin 2 (IL-2) in immune reconstitution was discussed by $\mathrm{Dr}$ Lane (plenary 30). IL-2 has the potential to maintain the CD4 lymphocyte pool by increasing proliferation of both naive and memory cell subsets. Theoretical concerns that HIV replication would also be enhanced remain but in vivo studies suggest that this effect is not significant and HIV viral load may even fall in the longer term as a result of preservation of CD4 cells.

In conclusion, the general direction of much of the material presented at the meeting was in favour of initial aggressive therapy in the great majority of patients. However, in the final plenary session (no 22) Professor I Weller cautioned the audience about adopting this approach uncritically, citing the following reasons: (1) doubts remain about the validity of $\mathrm{pVL}$ responses as surrogate markers in the long term; (2) current agents are unlikely to completely suppress viral replication leading to resistance and therapeutic failure; (3) this will be compounded by poor compliance; and (4) little is known about long term toxicity.

The Seventh European Conference on Clinical Aspects and Treatment of HIV Infection will take place in Lisbon, Portugal on 23-27 October 1999.- - K W RadclifFE, J D C Ross, Department of GU Medicine, Whittall Street Clinic, Whittall Street, Birmingham B4 6DH. 